\title{
Clear Cell Chondrosarcoma: Clinical Characteristics and Outcomes in 15 Patients
}

\author{
James H. Flint, ${ }^{1}$ Anthony P. Conley, ${ }^{2}$ M. Laura Rubin, ${ }^{3}$ Lei Feng, ${ }^{3}$ Patrick P. Lin, ${ }^{4}$ \\ Bryan Moon, ${ }^{4}$ Justin Bird, ${ }^{4}$ Robert L. Satcher, ${ }^{4}$ and Valerae O. Lewis ${ }^{4}{ }^{4}$ \\ ${ }^{1}$ Naval Medical Center San Diego, Department of Orthopedics, San Diego 92134, CA, USA \\ ${ }^{2}$ University of Texas MD Anderson Cancer Center, Department of Sarcoma Medical Oncology, Houston, TX, USA \\ ${ }^{3}$ University of Texas MD Anderson Cancer Center, Department of Biostatistics, Houston, TX, USA \\ ${ }^{4}$ University of Texas MD Anderson Cancer Center, Department of Musculoskeletal Oncology, Houston, TX, USA
}

Correspondence should be addressed to Valerae O. Lewis; volewis@mdanderson.org

Received 30 June 2020; Revised 17 November 2020; Accepted 1 December 2020; Published 30 December 2020

Academic Editor: Manish Agarwal

Copyright (c) 2020 James H. Flint et al. This is an open access article distributed under the Creative Commons Attribution License, which permits unrestricted use, distribution, and reproduction in any medium, provided the original work is properly cited.

Background. Clear cell chondrosarcoma (CCC) represents less than $6 \%$ of all chondrosarcomas, and thus, our understanding of this rare entity is limited. Analyzing clinical characteristics and treatment patterns, thus increasing our knowledge, may improve treatment strategy. We review our institutional experience with 15 patients, including one case with dedifferentiation. Methods. A retrospective review was conducted in CCC patients treated at our institution from 1996 to 2015, with at least 2-year follow-up. Descriptive statistics and Kaplan-Meier survival analyses were performed. Results. Of 19 patients identified, 15 patients had at least 2-year follow-up and were included. The median age at diagnosis was 43 years. $80 \%$ were male. The most common presenting signs were pain (12 patients; $80 \%$ ) and fracture (2 patients; $13.3 \%)$. The most common site was proximal femur (8 patients; $53 \%)$. All patients had MSTS Stage I disease. Primary treatment included wide resection in 10 patients (67\%) and intralesional or marginal resection in 5 patients (33\%). Three patients died of disease during the study period, 1 with dedifferentiation of recurrent CCC. The median time to death from disease was 15.3 years (95\% CI: (14.2; NA)). The median time to either recurrence or death was 7.73 years for patients who had intralesional/marginal resection and 16.44 years for patients with wide resection (HR (wide vs. intralesional $/$ marginal $)=0.21,95 \% \mathrm{CI}:(0.04 ; 1.18), p=0.053)$. The median time to recurrence or death was significantly shorter for patients not initially treated at a sarcoma center $(p=0.01)$. Conclusions. CCC is a rare entity, and our understanding of it is still evolving. We observed a higher recurrence rate for intralesional or marginal resection, and wide resection alone remains the mainstay of treatment. Better outcomes were observed in patients initially treated by trained musculoskeletal oncologists. Due to the propensity of CCC to recur decades after initial resection, lifelong surveillance is recommended.

\section{Introduction}

Clear cell chondrosarcomas (CCCs) are rare tumors, representing less than $6 \%$ of all chondrosarcomas [1-5]. Unlike conventional chondrosarcoma, they have a predilection for the epiphysis of long bones and can be mistaken for benign entities $[1,4,6-11]$. Wide resection alone is the treatment of choice, as chemotherapy and radiation are largely ineffective $[1-3,8,10,12,13]$. CCC is known for very late local and metastatic recurrence, and reports of dedifferentiated cases have also been described [1, 3, 4, 14-18].

The current literature is limited to a few small case series and multiple case reports. Thus, our understanding of this rare entity is still very limited. Analyzing clinical characteristics and treatment patterns, thus increasing our knowledge, may improve outcomes. We reviewed our institutional experience with 15 patients, including one unique case with dedifferentiation, and we aim to assess disease- 
specific and recurrence-free survival, as well as the impact of margin status on these outcomes.

\section{Materials and Methods}

A retrospective review of all CCC patients treated at MD Anderson Cancer Center from 1996 to 2015 was conducted, utilizing our Tumor Registry and Sarcoma Pathology Database. Patients with less than 2 years of follow-up were excluded. Resection margin was taken from the pathology report and described as $\mathrm{R} 0$ (negative, $>/=2 \mathrm{~mm}$ to closest margin), R1 (microscopically positive, within $2 \mathrm{~mm}$ to closest margin), or R2 (grossly positive margin). Descriptive statistics, as well as Kaplan-Meier survival analyses (diseasespecific and recurrence-free survival analyses), were performed. Specific comparative outcomes were analyzed based on location of initial treatment and type of surgical treatment performed.

Disease-specific survival (DSS) was defined as the time interval between date of initial surgery and date of death for patients who died of disease and was censored at the last follow-up date or date of death from other causes. Recurrence-free survival (RFS) was defined as the time interval between date of initial surgery and date of recurrence or disease-specific death, whichever occurred first, and was censored at the last follow-up date for patients who neither recurred nor died, or died from other causes. Survival curves were estimated using the Kaplan-Meier method and differences in survival among groups were assessed using twosided log-rank tests. Univariable Cox proportional hazards regression models were used to estimate effect sizes of risk factors. Statistical analysis was conducted in $\mathrm{R}$ version 3.4.2.

\section{Results}

Fifteen patients were included in this retrospective review, after excluding 4 additional patients with less than two years of follow-up. Median follow-up time was 104 months (95\% CI: (57; NA)). Median age at diagnosis was 43 years (range: 25 to 74 years). $80 \%$ were male. The initial presenting symptom/sign was pain (12 patients; $80 \%)$ or fracture (2 patients; $13 \%)$. The most common site of disease was proximal femur (8 patients; 53\%). 12 patients had tumors in the epiphyseal and/or metaphyseal location (80\%), the remainder being in the chest wall (2) and spine (1). All patients had MSTS Stage I disease at diagnosis. Primary treatment included wide resection in $10(67 \%)$ and intralesional or marginal resection in the remainder (5 patients, 33\%). Seven patients were treated at a nonsarcoma center initially, 5 of which were not biopsied prior to treatment, and the majority received intralesional treatment ( 5 of 7 , or $71 \%$ ). Six of these 7 patients either died or had recurrence $(86 \%)$. Clinical and treatment variables are summarized in Tables 1 and 2 .

3.1. Disease-Specific Survival. Three patients died of disease progression during the study period, and two others from non-disease-related conditions. 1 patient died after dedifferentiation of his recurrent CCC. The median time to death from disease was 15.3 years (95\% CI: (14.2; NA)). Diseasespecific survival for all subjects can be observed in Figure 1.

Disease-specific survival (DSS) was not significantly different between surgical treatment groups $(p>0.99)$. Tumor size $(<=8 \mathrm{~cm}$ vs. $>8 \mathrm{~cm})$ did not correlate with DSS $(p=0.23)$. Four of seven patients received chemotherapy for recurrence. Of these four, two (50\%) died of disease. Out of 3 patients with no chemotherapy after recurrence, 1 died of disease (33\%). The median DSS time was 15.3 for patients who did not receive chemotherapy for recurrence and 16.7 (95\% CI: (14.2; NA)) for patients with chemotherapy for recurrence.

3.2. Recurrence-Free Survival. Seven patients had recurred during the study period, three of which died of disease. 4 patients had local recurrence (LR) only, 2 had LR and distant metastasis (DM), and 1 had DM alone. The median time to recurrence or death was 12.67 years (95\% CI: (7.73; NA)). Only 1 of 2 patients initially presenting with pathologic fracture had recurrence. Two patients had recurrence beyond 10 years postop or died of disease (Figure 2).

Of those patients who recurred, four patients had intralesional/marginal resection performed and three had wide resection. The median time to recurrence or death was 7.73 years (95\% CI: $(4.49$; NA)) for patients who had intralesional/marginal resection, and 16.44 years (95\% CI: (8.39; NA)) for patients with wide resection (HR (wide vs. intralesional/marginal resection $)=0.21,95 \% \mathrm{CI}:(0.04 ; 1.18)$, $p=0.053$, Figure 3).

When considering initial treatment facility for patients who either recurred or died of disease, 1 had initial treatment at MD Anderson first and 6 had initial treatment outside of MD Anderson. The initial treatment at outside facilities consisted of curettage or marginal resection in 4 of the 6 cases $(67 \%)$. The median time to recurrence or death was 16.4 years for the patient treated at MD Anderson first and 7.73 years (95\% CI: $(4.49$; NA)) for patients treated at other facilities first $(p=0.01$, Figure 4$)$.

\section{Discussion}

Clear cell chondrosarcomas (CCCs) are rare tumors first described by Unni et al. in 1976 and represent less than 6\% of all chondrosarcomas [1-5]. Unlike conventional chondrosarcoma, CCC has a predilection for the epimetaphysis of long bones, making it difficult to differentiate from other entities such as carcinoma metastases, chondroblastoma, giant cell tumor, and aneurysmal bone cyst on the basis of imaging alone [1, 4, 6-11]. Radiographic findings are typically nonspecific, with X-rays showing a lytic lesion. To aid in narrowing the differential diagnosis, a thorough history and advancing imaging including magnetic resonance imaging (MRI) and computed tomography (CT) are helpful $[7,19,20]$.

Core needle biopsy is the diagnostic method of choice in most cases. Cytologic analysis via fine-needle aspiration can also be employed, although findings are typically not specific to CCC, and no definitive cytogenetic link has been 
TABLE 1: Clinical characteristics.

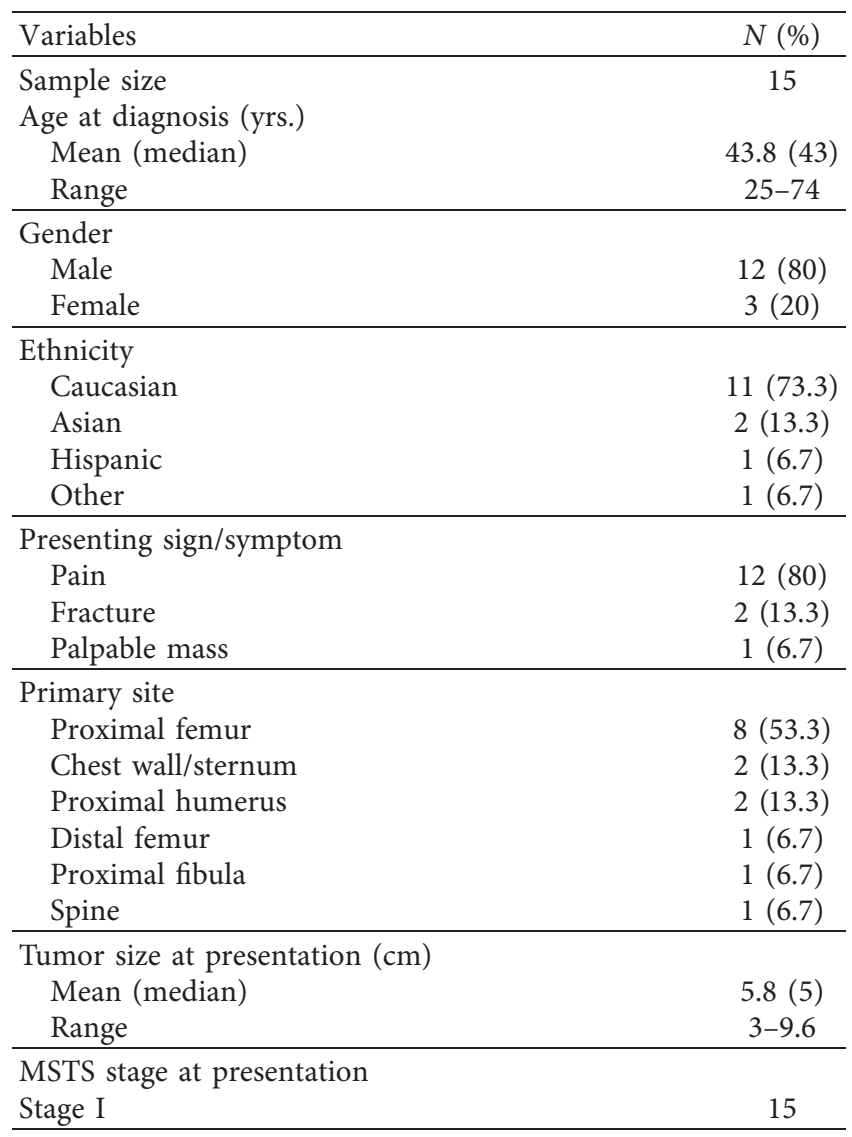

TABle 2: Treatment characteristics.

\begin{tabular}{lc}
\hline Variables & $N(\%)$ \\
\hline Biopsy prior to surgery & $9(60)$ \\
Yes & $6(40)$ \\
No & $10(66.7)$ \\
\hline Surgical management & $4(26.7)$ \\
$\quad$ Wide resection & $1(6.7)$ \\
Intralesional/curettage & \\
Marginal & $10(66.7)$ \\
Resection margins (as per pathologist report) & $2(13.3)$ \\
Free (R0) & $1(6.7)$ \\
Micro (R1) & $2(13.3)$ \\
Gross (R2) & \\
Unknown & $4(26.7)$ \\
\hline Recurrence status & $2(13.3)$ \\
Local only & $1(6.7)$ \\
Local and metastatic & $8(53.3)$ \\
Metastatic only & \\
None & $4(57.1)$ \\
\hline Chemotherapy after recurrence $(N=7)$ & $3(42.9)$ \\
Yes &
\end{tabular}

identified [21-23]. CCC is characterized by cells with distinct borders, central nuclei, and abundant clear vacuolated cytoplasm, surrounded by a cartilaginous matrix (Figure 5) $[1,2,5,20]$. Giant cells can also be present $[1,11,15,17]$.

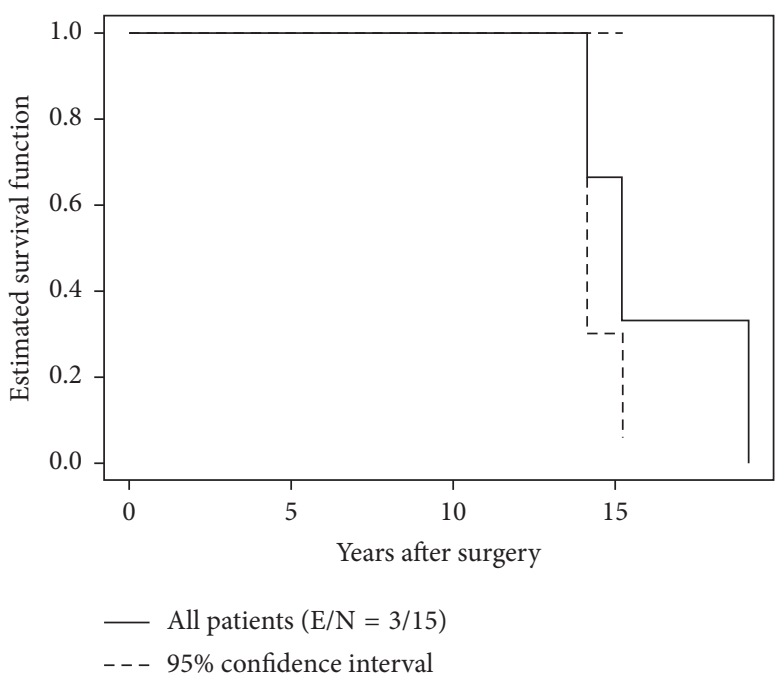

Figure 1: Disease-specific survival for patients with clear cell chondrosarcomas.

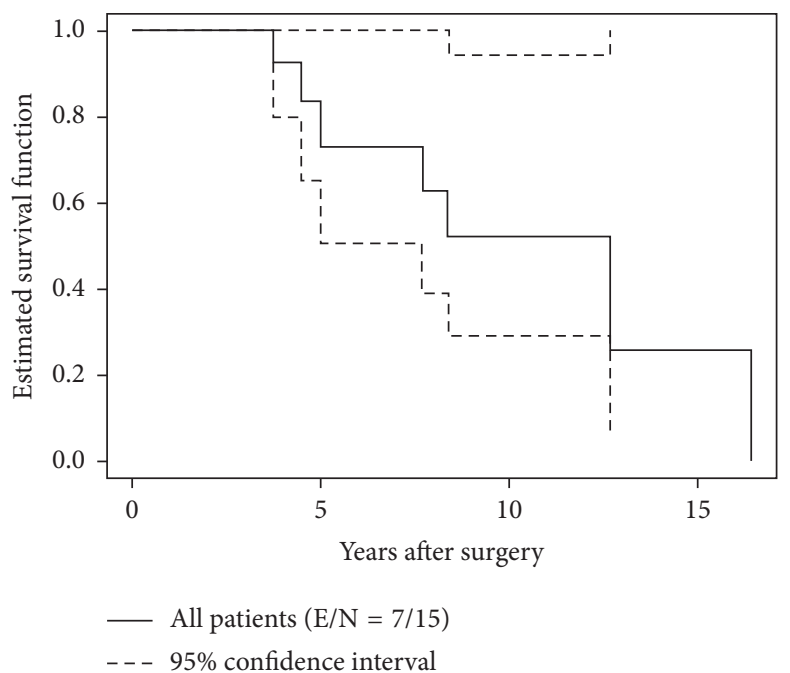

FIgURE 2: Recurrence-free survival. Note that two patients experienced recurrence or death beyond 10 years from initial resection.

Pathologic analyses of our cases were consistent, with all lesions demonstrating low-grade features.

Alkaline phosphatase (AP) has been described as a marker for primary and recurrent CCC. Ogose et al. concluded that CCC produce AP and levels decline or normalize after excision [24]. This finding was supported in a case series by Donati, who also showed decrease in AP postoperatively, as well as elevation of AP at the time of recurrence [12]. Ryu also reported normalization of AP postoperatively for a CCC of the sternum [25]. In the present study, AP was not a measured variable.

Chemotherapy is largely ineffective for chondrosarcomas, as is radiation, due to the slow-growing nature of the lesion. Italiano et al. evaluated the effect of chemotherapy on advanced chondrosarcomas, including two CCCs [13]. The majority (73\%) were treated with an anthracycline- 


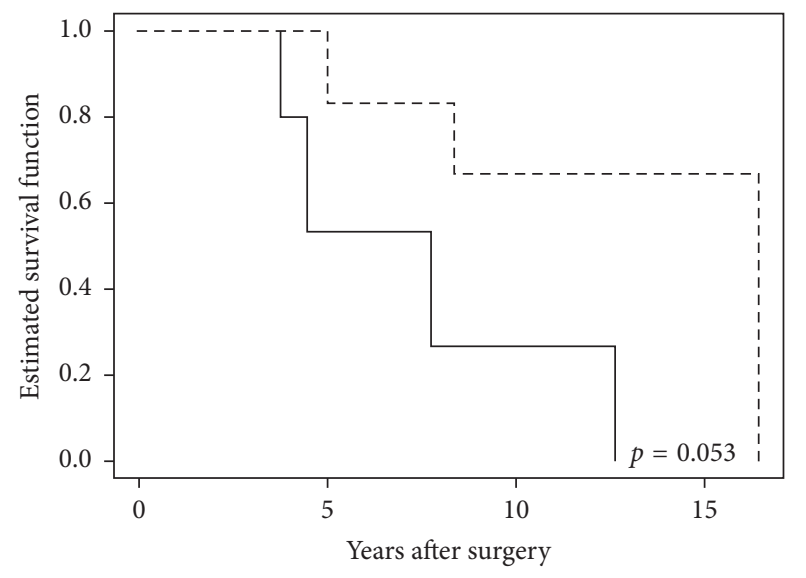

Type of surgery

— Intralesional/marginal resection $(\mathrm{E} / \mathrm{N}=4 / 5)$

- - - Wide resection $(\mathrm{E} / \mathrm{N}=3 / 10)$

FIgURE 3: Recurrence-free disease-specific survival by the type of resection performed. There was a significant association between wide resection and longer recurrence-free survival.

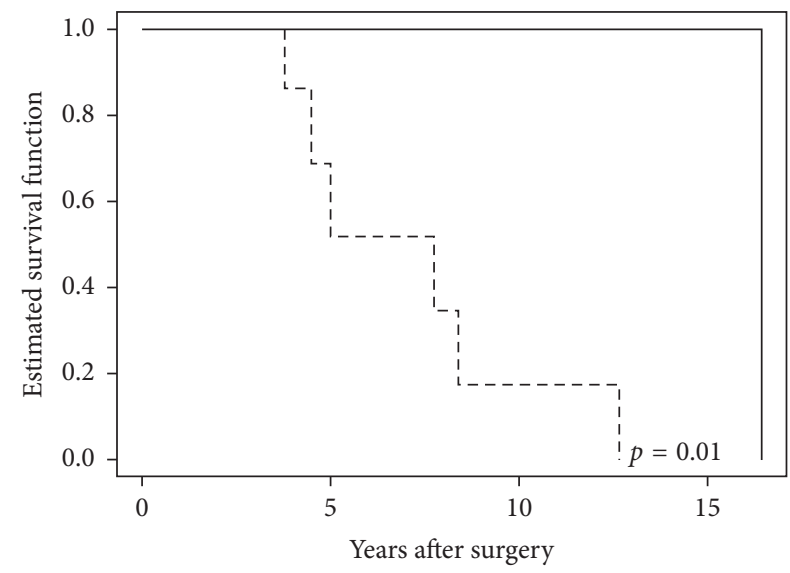

Treatment facility

- MD Anderson first ( $\mathrm{E} / \mathrm{N}=1 / 8)$

- - - Outside hospital first $(\mathrm{E} / \mathrm{N}=6 / 7)$

FIGURE 4: Recurrence-free survival by initial treatment center. Of the 8 patients that recurred during the follow-up period, 7 received initial care at outside facility first (nonsarcoma center), highlighting the importance of definitive treatment by sarcoma specialists.

based regimen. The two cases of CCC demonstrated 0\% objective response to chemotherapy. No patient in our study group received chemotherapy initially. Four of our cohort patients were administered chemotherapy after local or distant recurrence developed, as a salvage treatment. Two of the four (50\%) patients died during the follow-up period. One patient died of a myocardial infarction just prior to surgery to treat metastatic disease. One of the 3 patients with no chemotherapy after recurrence (33\%) died of disease. The median DSS time was 15.3 for patients who did not receive chemotherapy for recurrence and 16.7 (95\% CI: (14.2; NA)) for patients with chemotherapy for recurrence. The current

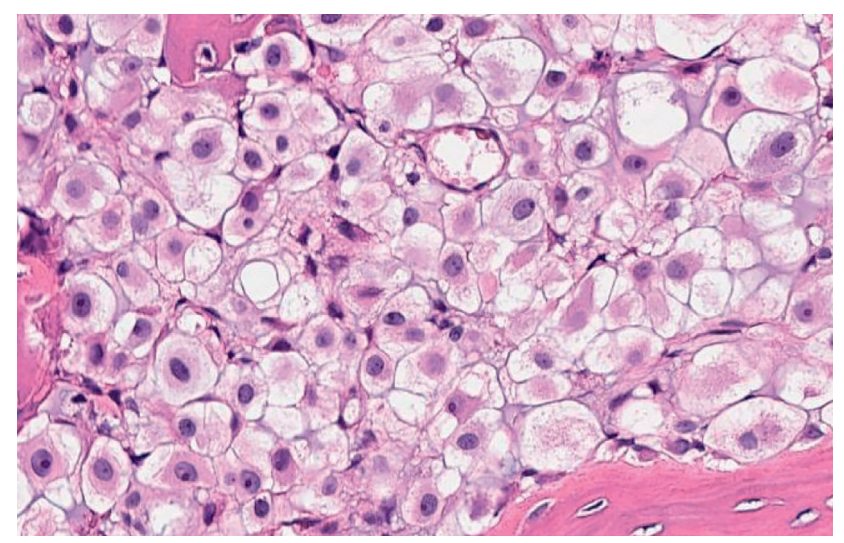

FIgURE 5: Clear cell chondrosarcoma. Cells show distinct borders, abundant cytoplasm, and central nuclei. These cells are located within the femoral head, with normal bone visible on this specimen.

study lacks sufficient power to definitively comment on the effectiveness of chemotherapy but, in general, supports that current chemotherapy regimens are not a proven effective treatment for CCC.

Regarding surgical management, wide resection is the treatment of choice $[1-3,10,12,13]$. It is our practice to treat all chondrosarcomas with wide resection alone. For patients referred acutely from outside facilities with a positive margin surgery, it is our general practice to subsequently perform wide resection. In metastatic cases, surgical treatment is considered as part of a multidisciplinary discussion, including options for chemotherapy and radiation. In a Mayo clinic study of 47 cases (the largest reported), only 2 patients treated with wide resection had recurrence [2]. In our cohort, 3 recurrences occurred after wide resection, all of which had negative (R0) margins intraoperatively. Diseasespecific survival was not significantly impacted by resection type. However, recurrence-free survival was marginally significant between groups, with a median time to recurrence or death of 8.7 years longer in the wide resection group. This may indicate that the biology of the tumor is an independent factor of disease-specific survival, particularly in light of the dedifferentiation of one of these tumors, a factor which needs to be explored further.

We specifically evaluated which patients received initial treatment at an outside facility; 7 were identified. 5 of the 7 patients were not biopsied before treatment, and $80 \%$ (4 out of 5) of these went on to receive intralesional or marginal resection. In fact, every patient that was initially treated with curettage or marginal resection came from an outside facility. All but one patient treated at an outside center first either died or had recurrence (6 of 7; 86\%). Eight patients were initially treated at our facility, and only one experienced recurrence of disease. This patient experienced significantly longer median RFS time (16.4 years versus 7.7 years; $p=0.01$ ). This highlights the critical importance of proper evaluation, diagnostic biopsy before surgery, and definitive treatment by trained musculoskeletal oncologists.

Four patients underwent open biopsy before initial treatment, two of which were performed at an outside 
hospital. The two with biopsies at the outside center both had recurrent disease, and both were proximal femur lesions. The two lesions with open biopsy at our center did not experience recurrence; one lesion was located in the proximal femur, and one in the fibula. While these numbers are small, it is important to note this difference. Unfortunately, details as to the approach for the biopsy (e.g., anterior vs lateral) are not available, but it is standard practice at our facility (and among sarcoma specialists in general) to perform the biopsy in line with the definitive surgical approach, such that the needle tract and skin can be resected with the specimen. While it is impossible to determine with certainty, this might explain the difference in recurrence among this subgroup.

Clear cell chondrosarcoma is known for late recurrence, and metastatic and dedifferentiated cases have also been described [1, 3, 4, 14-18]. Several authors report cases of recurrence beyond 19-year follow-up $[4,12,14,16]$. In the present study, two patients presented with late local recurrence ( $>10$ years): one at 12 years postoperatively and another at 16 years. This is consistent with prior studies, and we support the opinion that follow-up for CCC should extend life-long (we recommend annual imaging after 5 years of closer surveillance, per local algorithm). MRI can be employed for cases where large, metal implants are not needed for reconstruction. Otherwise, clinical and X-ray examination should be performed. Chest imaging is routinely recommended as well, which may include CT and/or chest X-ray, depending on presence or absence of pulmonary nodules.

Three patients in our cohort developed metastatic disease. Another patient developed dedifferentiated CCC after local recurrence and later developed metastases. Three of the four were treated via wide resection with negative margins. The fourth was initially treated via curettage with positive margins but revised to wide resection after presenting to our facility. The earliest metastatic disease presented 5 years postoperatively. Two of the three patients who developed metastatic disease were treated with adjuvant chemotherapy, with varying regimens for each. One patient was administered adjuvant chemotherapy after local recurrence of a spine lesion with dural involvement. Of the four patients in our cohort treated with chemotherapy, two of them died of disease. The remaining patients had bone-only metastatic disease to the spine or proximal femur and had been treated with pazopanib plus denosumab for the past 3 years of follow-up. This particular patient exhibited a decrease in FDG avidity on serial PET/CT imaging studies. There were no distinguishing characteristics identified that correlate with metastatic potential but, as aforementioned, may be related to unique tumor biology that is yet unknown. Since this was the only patient treated with pazopanib plus denosumab during the defined period of this study, it is not clear whether the therapeutic effect observed in this patient was a singular event or indicative of a true therapeutic effect upon this disease histology. Furthermore, it is unclear if this effect truly impacted his survivorship or the natural history of his disease despite metastasis.

The patient with dedifferentiation was a 53-year-old male with a right distal femur CCC. He underwent wide resection and limb salvage with an endoprosthesis. Local recurrence developed 16 years later, with dedifferentiation of CCC noted on histologic exam. He was treated with transfemoral amputation. One year later, he developed distant metastases to the lung, liver, and spine. Pathologic fracture of L4 was present, and he underwent palliative stabilization of his spine, followed by postoperative radiation and adjuvant doxorubicin-based chemotherapy. Despite this, he experienced progressive metastases, including to the brain, and he ultimately died of disease.

Overall survival for CCC is generally good. In the largest study to date (47 patients), Bjornsson et al. reported an overall mortality of $15 \%$ [2]. Itala et al. reported a ten-year overall survival of $89 \%$ and disease-free survival of $68 \%$ in 16 patients. Their study noted that the resection margin was the most significant factor affecting survival [3]. In our cohort, disease-specific mortality was $20 \%$. 10-year disease-free survival was $100 \%$, similar to these prior studies.

Limitations of this study include the inherent limits of retrospective study design. Some data were not collected in the database and, therefore, not available for analysis (i.e., alkaline phosphatase). There were 2 patients that did not have recent follow-up within two years of the end of the study period. This limits the power of statistical analysis with regard to disease-free survival.

Other limitations include selection bias, as there are likely cases of CCC that are treated at other facilities and never referred to a sarcoma center. Therefore, the true incidence of CCC is unknown, and statistical data need to be interpreted in light of this fact. Further selection bias exists in that cases referred from outside centers tend to be more complicated or associated with positive margins. Nearly half of the referred cases had a positive margin, and all patients who were treated with other than wide resection were referred from outside facilities. These sources of bias likely skewed the statistical interpretation.

\section{Conclusions}

In conclusion, CCC is a rare entity, and our understanding of it is still evolving. Although disease-specific survival was not significantly different, we observed a higher recurrence rate and a shorter recurrence-free interval for intralesional or marginal resection, and we continue to support wide resection alone as the mainstay of treatment. Better outcomes were observed in patients initially treated by trained musculoskeletal oncologists. Whenever possible, prompt referral to sarcoma specialists should follow suspicion or confirmation of this diagnosis with appropriate biopsy. Due to the propensity of clear cell chondrosarcoma to recur decades after initial resection, lifelong surveillance is recommended. 


\section{Data Availability}

The data in this study were derived from patient records at our institution and are thus not available for public release. All pertinent data are published in the accompanying tables and figures.

\section{Disclosure}

The level of evidence is retrospective review, Level IV.

\section{Conflicts of Interest}

The authors declare that there are no conflicts of interest existing in this study.

\section{References}

[1] K. S. Ayoub, R. J. Grimer, S. R. Carter, D. C. Mangham, A. M. Davies, and R. M. Tillman, "Clear cell chondrosarcoma of bone," Sarcoma, vol. 3, no. 2, pp. 115-119, 1999.

[2] J. Bjornsson, K. K. Unni, D. C. Dahlin, J. W. Beabout, and F. H. SIM, "Clear cell chondrosarcoma of bone," The American Journal of Surgical Pathology, vol. 8, no. 3, pp. 223-230, 1984.

[3] A. Itälä, T. Leerapun, C. Inwards, M. Collins, and S. P. Scully, "An institutional review of clear cell chondrosarcoma," Clinical Orthopaedics and Related Research, vol. 440, no. \&NA, pp. 209-212, 2005.

[4] M. Laitinen, J. Nieminen, and T.-K. Pakarinen, "An unusual case of clear cell chondrosarcoma with very late recurrence and lung metastases, 29 years after primary surgery," Case Reports in Orthopedics, vol. 2014, Article ID 109569, 6 pages, 2014.

[5] K. K. Unni, D. C. Dahlin, J. W. Beabout, and F. H. SIM, "Chondrosarcoma," The Journal of Bone \& Joint Surgery, vol. 58, no. 5, pp. 676-683, 1976.

[6] M. Campanacci, F. Bertoni, and M. Laus, "Clear cell chondrosarcoma," Italian Journal of Orthopaedics and Traumatology, vol. 6, no. 3, pp. 365-372, 1980.

[7] M. S. Collins, T. Koyama, R. G. Swee, and C. Y. Inwards, "Clear cell chondrosarcoma: radiographic, computed tomographic, and magnetic resonance findings in 34 patients with pathologic correlation," Skeletal Radiology, vol. 32, no. 12, pp. 687-694, 2003.

[8] N. S. Datt, C. N. S. Mounika, K. R. Kiran, D. R. Rao, and V. Sandeep, "Clear cell chondrosarcoma proximal femur with secondary aneurysmal component-a rarity," Journal of Clinical Orthopaedics and Trauma, vol. 8, no. 1, pp. 93-95, 2017.

[9] A. H. Kaim, R. Hügli, H. M. Bonél, and G. Jundt, "Chondroblastoma and clear cell chondrosarcoma: radiological and MRI characteristics with histopathological correlation," Skeletal Radiology, vol. 31, no. 2, pp. 88-95, 2002.

[10] D. Present, P. Bacchini, G. Pignatti, P. Picci, F. Bertoni, and M. Campanacci, "Clear cell chondrosarcoma of bone. A report of 8 cases," Skeletal Radiology, vol. 20, no. 3, pp. 187-191, 1991.

[11] T. Tay, B. Wong, K. Sittampalam, and T. Lie, "Clear cell chondrosarcoma with secondary aneurysmal bone cyst changes," Singapore Medical Journal, vol. 55, no. 3, pp. e49-51, 2014.
[12] D. Donati, J.-Q. Yin, M. Colangeli et al., "Clear cell chondrosarcoma of bone: long time follow-up of 18 cases," Archives of Orthopaedic and Trauma Surgery, vol. 128, no. 2, pp. 137-142, 2008.

[13] A. Italiano, O. Mir, A. Cioffi et al., "Advanced chondrosarcomas: role of chemotherapy and survival," Annals of Oncology, vol. 24, no. 11, pp. 2916-2922, 2013.

[14] L. Bagley, J. B. Kneeland, M. K. Dalinka, P. Bullough, and J. Brooks, "Unusual behavior of clear cell chondrosarcoma," Skeletal Radiology, vol. 22, no. 4, pp. 279-282, 1993.

[15] D. Corradi, P. Bacchini, N. Campanini, and F. Bertoni, "Aggressive clear cell chondrosarcomas: do distinctive characteristics exist? A report of 4 cases," Archives of $\mathrm{Pa}$ thology \& Laboratory Medicine, vol. 130, no. 11, pp. 16731679, 2006.

[16] D. Hartwright, D. C. Mangham, and R. J. Grimer, "Reactivation of tumor after 19 years? A case of local recurrence in clear cell chondrosarcoma," International Orthopaedics, vol. 24, no. 2, pp. 112-114, 2000.

[17] R. K. Kalil, C. Y. Inwards, K. K. Unni et al., "Dedifferentiated clear cell chondrosarcoma," The American Journal of Surgical Pathology, vol. 24, no. 8, pp. 1079-1086, 2000.

[18] M. Manfrini, S. Fiscina, A. Righi, J. M. Montes, and D. Vanel, "Multiple or metastatic clear cell chondrosarcoma: a case report," Clinical Sarcoma Research, vol. 4, p. 12, 2014.

[19] H. Douis and A. Saifuddin, "The imaging of cartilaginous bone tumours. II. Chondrosarcoma," Skeletal Radiology, vol. 42, no. 5, pp. 611-626, 2013.

[20] S. Elojeimy, W. A. Ahrens, B. Howard et al., "Clear-cell chondrosarcoma of the humerus," Radiology Case Reports, vol. 8, no. 2, p. 848, 2013.

[21] X. S. Jiang, L. Pantanowitz, M. M. Bui, R. Esther, D. Budwit, and L. G. Dodd, "Clear cell chondrosarcoma: cytologic findings in six cases," Diagnostic Cytopathology, vol. 42, no. 9, pp. 784-791, 2014.

[22] K. E. McHugh, C. L. Emory, G. E. Parks, and S. E. Kilpatrick, "Fine needle aspiration biopsy diagnosis of primary clear cell chondrosarcoma: a case report," Diagnostic Cytopathology, vol. 46, no. 2, pp. 165-169, 2018.

[23] J. Nishio, J. D. Reith, A. Ogose, G. Maale, J. R. Neff, and J. A. Bridge, "Cytogenetic findings in clear cell chondrosarcoma," Cancer Genetics and Cytogenetics, vol. 162, no. 1, pp. 74-77, 2005.

[24] A. Ogose, T. Hotta, H. Kawashima et al., "Elevation of serum alkaline phosphatase in clear cell chondrosarcoma of bone," Anticancer Reserach, vol. 21, pp. 649-655, 2001.

[25] K. M. Ryu, N.-H. Myung, and P. W. Seo, "Clear cell chondrosarcoma arising from the sternum: a rare tumor in an uncommon location," The Annals of Thoracic Surgery, vol. 93, no. 5, pp. 1725-1727, 2012. 